\title{
Medición de humedad en suelos: Revisión de métodos y características
}

\section{Soil Moisture Measurement: Review of Methods and Characteristics}

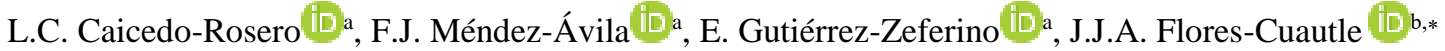 \\ a Maestría en Ingeniería Electrónica, TecNM, I.T. Orizaba, Oriente 9, Emiliano Zapata, Orizaba, Ver. México. \\ ${ }^{b}$ CONACyT-TecNM, I.T. Orizaba, Oriente 9, Emiliano Zapata, Orizaba, Ver. México.
}

\begin{abstract}
Resumen
El agua es un recurso prioritario de estudio en varias ramas de la ciencia, incrementado por las dinámicas sociales. Es un factor determinante en la fertilidad del suelo, afectando el desarrollo de los cultivos, su disponibilidad da sustento a los procesos biológicos; también ayuda a regular la temperatura del suelo. En la actualidad se busca monitorear la cantidad de agua disponible, para optimizar su uso y controlar las propiedades del suelo. En el presente trabajo se hace una revisión de las propiedades eléctricas del suelo a partir del uso de diferentes métodos e instrumentos para la medición de la humedad. Los instrumentos para medición de humedad basados en propiedades eléctricas son fácilmente adaptables para ser automatizados y operados en forma remota. Este tipo de instrumentos, aunque requieren de mantenimiento y calibración, poseen buena precisión y un corto tiempo de muestreo, lo que da factibilidad a su aplicación a las ciencias agrícolas.
\end{abstract}

\section{Palabras Clave:}

Humedad del suelo, instrumentos de medición, metodologías de medición.

\begin{abstract}
Water is a priority resource for study in various branches of science, increased by social dynamics. In the earth sciences, it is a determining factor in soil fertility affecting crop development; its availability supports biological processes; it also helps regulate soil temperature and greenhouse gas retention. Nowadays, the aim is monitoring the amount of water available for optimizing its use and soil properties. In the present work, a review of different methods and instruments for measuring soil moisture using the electrical properties is made. These instruments are easily adaptable to be automated and remote measurements. However, they require maintenance and calibration, they have good accuracy, and their sampling time is fast, which gives feasibility to their application to agricultural sciences that require continuous measurements in difficult access areas.
\end{abstract}

Keywords:

Soil moisture, measurement methods, measurement instruments.

\section{Introducción}

Las propiedades del suelo se han analizado desde diversos campos de la ciencia como en ingeniería, geología, ecología y agricultura, empleando diferentes técnicas de análisis (Cruz et al., 2004). El análisis de los nutrientes presentes en el suelo se determina siguiendo procedimientos ya estandarizados (Motsara \& Roy, 2008). La humedad en el suelo puede ser un factor de riesgo de deslizamientos cuando la humedad se combina con la presencia de arcillas de tipo alófanas (Ramirez, 2009) debido a sus características tixotrópicas, y deficiencias en la consolidación del suelo (Guzzetti et al., 2020). El contenido de humedad en el suelo (CHS) se encuentra relacionado con la retención de metano atmosférico dentro de los sistemas forestales (Seneviratne et al., 2010), (Liu et al., 2019). El conocimiento de los diferentes métodos de medición de humedad en suelos, así como el análisis de las ventajas y desventajas de cada uno de los métodos analizados en el presente trabajo puede servir en la elección del método de medición acorde al tipo de muestra.

\section{Estructura y composición del suelo}

El suelo es una mezcla de minerales primarios y secundarios (coloides inorgánicos) y materia orgánica (MO) que determinan el CHS. La superficie específica depende directamente de los coloides del suelo, arcilla y MO del suelo, lo que influye en la

\footnotetext{
*Autor para la correspondencia: jflores_cuautle@hotmail.com Gutiérrez Zeferino), jflores_cuautle @ hotmail.com (José de Jesús Agustín Flores Cuautle)

Correo electrónico: 1caicedo333@hotmail.com (Luis Carlos Caicedo Rosero), fabriciomendeza@gmail.com (Fabricio de Jesús Méndez Ávila), erickgzeferino@gmail.com (Erick 
retención de agua, a mayor superficie específica, mayor CHS. La superficie específica se define como la relación área-peso entre partículas $\left(\mathrm{m}^{2} / \mathrm{g}\right)$ por lo que no depende de la granulometría ni de la compactación del suelo. Por tanto, en la determinación del agua presente en el suelo intervienen tanto propiedades fisicoquímicas como estructurales, lo que explica la variabilidad del CHS. En la Tabla 1 se muestran las principales características físicas y químicas de los tipos de suelo, su condición higroscópica y la fuerza principal para el CHS según su composición.

Las características morfológicas y constitutivas afectan el CHS, cristales de átomos de mayor diámetro favorecen la formación de hidratos en tamaños de partículas menores, así como la presencia de material orgánico en limos también aumenta a la retención de agua (Garay Diaz et al., 2007).

Tabla 1. Tipos de suelo y materiales constituyentes (Juárez Badillo \& Rico Rodríguez, 2010).

\begin{tabular}{|c|c|c|c|c|c|}
\hline \multicolumn{2}{|c|}{ Tipo de suelo } & \multirow{2}{*}{$\begin{array}{c}\text { Compuesto } \\
\text { Mayormente } \\
\text { cuarzo y } \\
\text { cuarcita. En } \\
\text { menor cantidad } \\
\text { caliza, basalto, } \\
\text { granito y } \\
\text { dolomita. }\end{array}$} & \multirow{2}{*}{$\begin{array}{c}\text { Higroscopía } \\
\text { Baja. } \\
\text { Retención } \\
\text { superficial. }\end{array}$} & \multirow{2}{*}{$\begin{array}{c}\begin{array}{c}\text { Diámetro } \\
\text { de }\end{array} \\
\text { partícula } \\
(\mathrm{mm}) . \\
2-64\end{array}$} & \multirow{2}{*}{$\begin{array}{c}\text { Fuerza } \\
\text { Principal - } \\
\text { (Proceso) }\end{array}$} \\
\hline \multirow{2}{*}{ Grueso } & Gravas & & & & \\
\hline & Arenas & $\begin{array}{c}\text { Sílice, cuarzo, } \\
\text { caliza molida, } \\
\text { en menor } \\
\text { cantidad } \\
\text { hierro, } \\
\text { feldespato o } \\
\text { yeso. }\end{array}$ & $\begin{array}{c}\text { Baja. } \\
\text { Retención } \\
\text { superficial. }\end{array}$ & $\begin{array}{c}6.25 \times 10^{-2} \\
-2\end{array}$ & $\begin{array}{c}\text { Gravedad y } \\
\text { Electrostática } \\
\text { (Fisisorción) }\end{array}$ \\
\hline \multirow[b]{2}{*}{ Fino } & Limos & $\begin{array}{c}\text { Compuestos } \\
\text { orgánicos, en } \\
\text { menor cantidad } \\
\text { óxido de } \\
\text { hierro, calcitas } \\
\text { y feldespatos. }\end{array}$ & $\begin{array}{c}\text { Media. } \\
\text { Retención } \\
\text { por enlace } \\
\text { químico. } \\
\text { Formación } \\
\text { de hidratos. }\end{array}$ & $\begin{array}{l}3.9 \times 10^{-3} \\
6.25 \times 10^{-2}\end{array}$ & $\begin{array}{c}\text { Electrostática } \\
\text { (Quimisorción) }\end{array}$ \\
\hline & Arcillas & $\begin{array}{c}\text { Filosilicatos de } \\
\text { aluminio } \\
\text { hidratados, en } \\
\text { menor cantidad } \\
\text { hierro, } \\
\text { magnesio, } \\
\text { metales } \\
\text { alcalinos, } \\
\text { tierras alcalinas } \\
\text { y otros } \\
\text { cationes. }\end{array}$ & $\begin{array}{c}\text { Alta. } \\
\text { Retención } \\
\text { por enlace } \\
\text { químico. } \\
\text { Formación } \\
\text { de hidratos. }\end{array}$ & $\underset{3}{<3.9 \times 10^{-}}$ & $\begin{array}{c}\text { Electrostática } \\
\text { (Quimisorción) }\end{array}$ \\
\hline
\end{tabular}

\subsection{Tipos de humedad}

La humedad se define como la cantidad de agua contenida en un líquido o sólido por adsorción o absorción que puede eliminarse sin alterar sus propiedades químicas (Fraden, 2016), con relación al suelo, se ha determinado que no existe un solo tipo de humedad, sino que depende de las fuerzas que actúan sobre las moléculas de agua, que están determinadas por la estructura y composición del suelo. La humedad libre es la fracción que no está sujeta a fuerzas moleculares, esto es puede desplazarse hacia capas profundas como consecuencia de la gravedad por lo cual esta fracción no es disponible para las plantas. Briggs y Terzaghi (S.U. et al., 2014) analizaron y concluyeron que existen tres tipos de humedad:

Humedad gravitacional: humedad libre, que se mueve debido a la fuerza de la gravedad, se encuentra en los macroporos, de movimiento rápido, en suelos bien drenados y no se considera humedad disponible. Drena en 2-3 días después de la lluvia dependiendo de la unidad del suelo.
Humedad capilar: disponible como humedad de microporo y es responsable de todas las interacciones fisicoquímicas y mineralógicas-biológicas entre el suelo y el medio ambiente. Se mantiene dentro del suelo debido a la cohesión y adhesión contra la fuerza de la gravedad

Humedad higroscópica: película muy delgada alrededor de la superficie de las partículas del suelo, es muy difícil de eliminar debido a la presencia de fuerzas de adhesión. El suelo arcilloso retiene más cantidad de humedad higroscópica (denominada agua estructural) en comparación con la arena.

La capacidad de retención de agua se suele estimar como el agua retenida en el suelo después de drenar el exceso de agua gravitacional (humedad libre), pero no necesariamente disponible para las plantas, en tanto que el punto de marchitamiento permanente $(0.33 \mathrm{~atm})$ se define como el contenido de agua del suelo debajo del cual las plantas comienzan a marchitarse. El agua disponible para las plantas es aquella que puede ser absorbida por sus raíces (Domingo-Santos et al., 2008).

Buckingham y Gardner en (S.U. et al., 2014) desarrollaron una curva característica del agua del suelo o curva de retención de humedad del suelo, que es una relación entre el contenido de humedad y la capacidad de succión del suelo (potencial de humedad del suelo) único para cada tipo, que depende de su nivel de energía. Se utiliza para predecir el almacenamiento de humedad del suelo, la capacidad de campo (1.3 atm), el punto de marchitez y es útil para fines de riego. También se puede emplear para comprender las características de secado y humectación del suelo y su estructura de poros (Liu et al., 2019).

Se ha planteado la teoría de la doble capa catiónica (Juárez Badillo \& Rico Rodríguez, 2010) para explicar el comportamiento de las moléculas de agua y el CHS como se ilustra en la Figura 1. La alta electronegatividad del oxígeno genera cargas estáticas positivas en la superficie de los cristales de óxidos y sales, que a la vez inducen un dipolo en las moléculas de agua circundantes, aumentando así las fuerzas atractivas y consecuentemente su retención. La magnitud de las fuerzas de atracción hacia el agua depende de las características geométricas y estructurales del suelo, tal como se establece en la Tabla 1, a menor diámetro de partícula mayor superficie específica y mayor intensidad en las fuerzas de atracción moleculares, pudiendo generar compuestos hidratados, que dependen del tamaño y tipo de catión.

La atracción cristalina hace que pueda llegar a incluirse dentro de su estructura una o varias moléculas de agua, soliéndose llamar agua sólida, sin ser un término preciso. Las fuerzas de atracción cristal - agua disminuyen rápidamente a medida que aumenta la distancia hacia la superficie del cristal (Verwey, 1947) presentando un punto de cambio de comportamiento, tal vez por acumulación de cargas positivas alrededor del cristal, donde las moléculas de agua están más libres, formando así la doble capa. La capa contigua corresponde con agua líquida fuertemente enlazada al cristal, la capa externa suele corresponder a agua en estado vapor, que se pierde fácilmente.

El valor límite disminuye en la presencia de iones disueltos en el agua (Figura 1) y también por la presencia de cristales contiguos al aportar cargas repulsivas; existen otros factores para los cuales aún no está determinada su relevancia en el contenido de humedad del suelo (D. A. Robinson et al., 2008). 


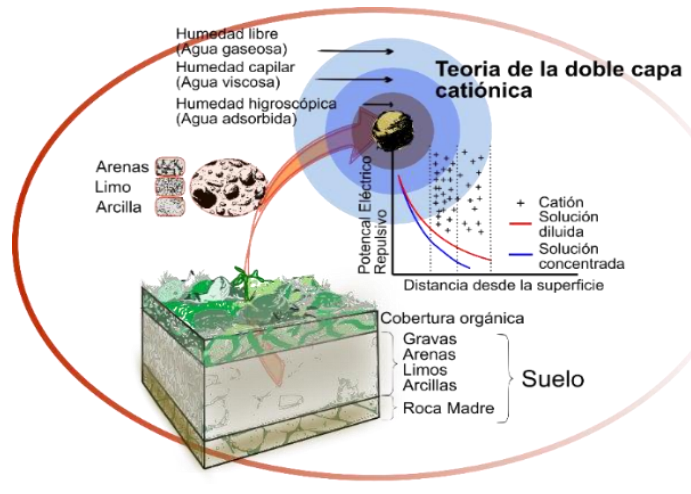

Figura 1. Teoría de la doble capa catiónica y la relación con las fuerzas atomicas (Elaboración propia basado en Juárez Badillo \& Rico Rodríguez, 2010)

Existen diferentes tipos de métodos y técnicas para el monitoreo del CHS (S.U. et al., 2014), (D. A. Robinson et al., 2008), (Berney \& Kyzar, 2012), (Dobriyal et al., 2012), (Robock et al., 2000), (Schmugge et al., 1980), (Walker et al., 2004), (Zazueta \& Xin, 1994). En este estudio se hace una revisión de los métodos que utilizan las propiedades eléctricas como medida indirecta del CHS.

\section{Propiedades eléctricas}

Las propiedades eléctricas del suelo son ampliamente utilizadas para determinar el CHS; entre las técnicas más comunes se pueden mencionar: Resistividad, Capacitancia, Reflectometría en el Dominio del Tiempo (TDR), y Reflectometría en el Dominio de la Frecuencia (FDR). Por otra parte, es posible utilizar la conductividad eléctrica del suelo para medir el CHS (Fraden, 2016), siendo el agua pura un mal conductor de electricidad, es la concentración de iones presente en el agua la que permite la conducción de la corriente eléctrica por lo tanto la conductividad es una función recíproca de resistividad específica.

\subsection{Técnica de sensor de resistividad del suelo}

El contenido de humedad del suelo se mide a través de la variación en la resistividad del suelo por medio de sondas (electrodos) que son enterrados en el suelo. En los sensores resistivos el valor absoluto de la resistividad del suelo depende de la concentración de iones, así como de la concentración de humedad. En este método se usan electrodos metálicos para medir la resistencia del suelo. Sin embargo, este método no es $100 \%$ recomendable para largos periodos de medición, o aplicaciones en que se requiera alta precisión, ya que la corriente continua causa la polarización del electrodo que cambia la resistencia de la interfaz electrodo-suelo, dicho cambio resulta en desviaciones en la medición después de un uso prolongado, por esta razón se recomienda limpiar los electrodos después de cada uso.

Como una alternativa a los electrodos metálicos, se pueden usar algunos materiales porosos como el nylon, la fibra de vidrio o el yeso, de esta forma, la resistencia eléctrica que presentan estos materiales estarán relacionada con la cantidad de humedad presente.

Lo anterior se resume en una simple analogía, mientras menos humedad esté presente en un suelo, su resistencia eléctrica aumenta, por ello este tipo de dispositivos son muy prácticos a la hora de determinar la cantidad de agua que hace falta aplicar en un suelo determinado o un área en específico (Rêgo Segundo et al., 2015), (Florentino, 2006). Tal como se mencionó anteriormente, el CHS depende de la estructura, composición y destinación de uso del suelo, cada cultivo necesita una cantidad total de agua diferente, que es la requerida para la evapotranspiración y la construcción celular, desde la plantación hasta la cosecha, según el régimen climático.

Tabla 2. Ventajas y limitaciones de los sensores de bloque de yeso (Florentino, 2006).

\begin{tabular}{ll}
\multicolumn{1}{c}{ Ventajas } & \multicolumn{1}{c}{ Limitaciones } \\
\hline $\begin{array}{l}\text { El efecto buffer sobre la salinidad del suelo es } \\
\text { hasta } 6 \mathrm{dS} / \mathrm{m} .\end{array}$ & $\begin{array}{l}\text { Depende de la temperatura (sufre } \\
\text { cambios) y presenta histéresis en la } \\
\text { medición. }\end{array}$ \\
$\begin{array}{l}\text { Las lecturas sobre la humedad del suelo son } \\
\text { directas. }\end{array}$ & $\begin{array}{l}\text { Es inservible en suelos muy agrietados } \\
\text { o arenosos. }\end{array}$ \\
$\begin{array}{l}\text { De fácil implementación y bajo costo. } \\
\text { Para el caso de la planificación del riego son una baja resolución. } \\
\text { muy requeridos. }\end{array}$ & Necesita de calibración frecuente. \\
\hline
\end{tabular}

\subsection{Sensores de matriz granular}

Este dispositivo se compone de un material granular de cuarzo, que envuelve a los electrodos que lo acompañan, éstos se encuentran rodeados por una membrana sintética y una malla protectora de acero inoxidable. Este tipo de sensores permiten una lectura de humedad más prolongada (periodos más largos de tiempo); la principal desventaja la baja resolución a niveles bajos de humedad, así mismo presenta tiempos de reacción lentos. Sus rangos de lectura son de -10 a -300 kPa (Muñoz-Carpena, 2004). Un resumen de las características de los sensores de matriz granular se da en la Tabla 3.

Tabla 3. Ventajas y limitaciones de los sensores de matriz granular (Florentino, 2006).

\begin{tabular}{ll}
\hline \multicolumn{1}{c}{ Ventajas } & \multicolumn{1}{c}{ Limitaciones } \\
\hline Diseño simple y bajo costo. & $\begin{array}{l}\text { Se ve afectado por la temperatura, por } \\
\text { lo que requiere calibración. }\end{array}$ \\
No presenta problemas de disolución & Baja resolución. \\
$\begin{array}{l}6 \mathrm{dS} / \mathrm{m}^{-1} \text { es lo máximo que se puede controlar } \\
\text { el efecto de salinidad. }\end{array}$ & $\begin{array}{l}\text { Su tiempo de reacción a la humedad } \\
\text { presente es muy lenta (Florentino, } \\
2006) .\end{array}$ \\
\hline
\end{tabular}

\subsection{Sensores genéricos de muy bajo costo: YL100, YL69}

Otros ejemplos de sensores de tipo resistivo para la medición de humedad presente en el suelo son los modelos comerciales YL100 y YL69; este tipo de sensores constan de dos superficies conductoras separadas por una distancia constante, las superficies conductoras se encuentran en contacto físico con el suelo que se desea medir. La resistencia en la interfaz sensor-suelo varía en función de la humedad presente en el suelo. Aunado a lo anterior, este tipo de dispositivos cuentan con un módulo electrónico que se encarga de medir la variación de voltaje que se presenta al haber una variación en la resistencia de las sondas anteriormente mencionadas. Sin embargo, al igual que cualquier dispositivo, se ve influenciado o afectado por factores externos, siendo en este caso la concentración de iones el que afecta la resistividad del suelo, debido a lo anterior es necesaria una constante calibración para una medición eficaz. Las categorías de cantidad de humedad presente en el suelo registradas por estos modelos de sensores resistivos se clasifican normalmente en seco, medio seco o medio húmedo, húmedo, muy húmedo (Farahani et al., 2014),(Adla et al., 2020). 


\subsection{Cálculo del CHS}

Dado que estos métodos están basados puramente en la resistencia eléctrica, el CHS se puede calcular a partir de un divisor de voltaje que se forma a partir de una resistencia de valor conocido (R) en serie con resistencia del suelo (RS), el voltaje de salida representa el CHS. Dado que el valor de la resistencia conocida, así como el voltaje aplicado pueden variar se requiere una calibración cuidadosa para estas técnicas.

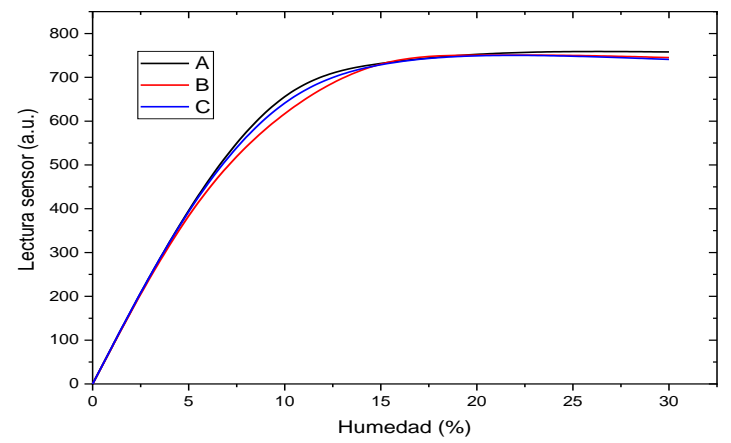

Figura 2. Curva de Calibración típica para el sensor resistivo (elaboración propia con datos de un sensor YL100)

De la Figura 2 es posible apreciar que este tipo de sensores presentan una alta sensibilidad para CHS menor al $12 \%$, para porcentajes de humedad superiores a este valor la sensibilidad del sensor disminuye.

\subsection{Técnica de reflectometría en el dominio del tiempo (TDR)}

Se basa en la relación entre las propiedades dieléctricas del suelo y su contenido de agua, debido a la diferencia entre la constante dieléctrica del suelo seco $(\approx 2.9)$ y el agua pura $(\approx 81)$. La permitividad del suelo se mide al determinar el tiempo de ida y vuelta de un pulso electromagnético que se propaga a lo largo de una guía, en forma de sonda insertada en el suelo, la velocidad del pulso es inversamente proporcional al CHS.

Esta técnica proporciona el contenido volumétrico de humedad del suelo es de alta precisión, aunque de un costo elevado, de bajo alcance espacial y una alta resolución temporal (Dobriyal et al., 2012), es una técnica no destructiva, de mediciones rápidas (aproximadamente $28 \mathrm{~s}$ ) y repetibles (S.U. et al., 2014). La sonda suele constar de dos o tres cilindros de acero inoxidable, tiene una aplicabilidad limitada en suelos altamente salinos (Dobriyal et al., 2012) pero para suelos salinos secos presenta buenas mediciones (S.U. et al., 2014), y se ve influenciada por la profundidad a la que se toman las muestras (Sinha et al., 2017).

\subsection{Cálculo del CHS}

La medición del CHS por medio de las propiedades eléctricas utiliza la permitividad o constante dieléctrica aparente del suelo la cual posee dos componentes, real e imaginaria, y depende de la vibración o relajación atómica y molecular de los componentes del suelo, lo que genera pérdida de energía. Tal como se observa en la Figura 3, la relajación depende de la frecuencia de excitación, para el agua presente en el suelo sus efectos se observan a partir de una frecuencia de $10 \mathrm{MHz}$.

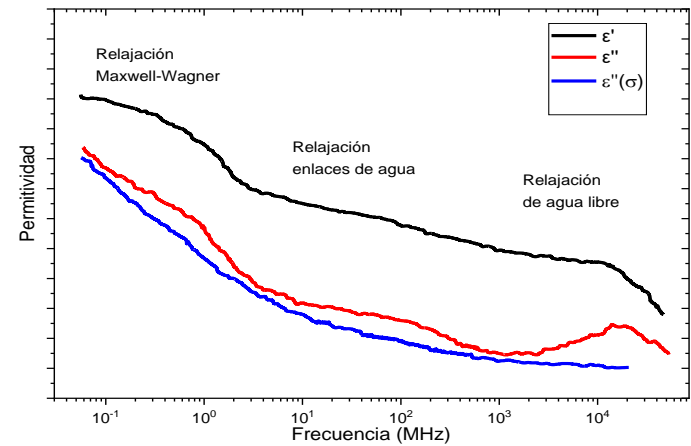

Figura 3. Propiedades dieléctricas del suelo dependientes de la frecuencia, parte real $\left(\varepsilon^{\prime}\right)$, imaginaria $\left(\varepsilon^{\prime}\right) y$ conductividad DC $\left(\varepsilon^{\prime \prime}(\sigma)\right)$ (van Dam et al., 2005)

En general las mediciones mediante TDR poseen buena precisión, aunque requieren calibración para evitar errores, asî como la revisión y mantenimiento de las sondas cuando se requiere hacer medidas constantes. Se reportan errores del $\pm 1 \%$ (Dobriyal et al., 2012) al $\pm 10 \%$ (Bobrov et al., 2019) cuando no se realiza una calibración previa. Bobrov y colaboradores 2019 estudiaron la influencia de los diferentes factores en este tipo de mediciones, encontrando que, para el análisis con curvas de calibración previa el error puede ser de hasta el $4 \%$. Se recomienda mediciones a una frecuencia entre $0.5-3 \mathrm{GHz}$ para mejorar la precisión de las medidas, permitiendo ampliar el espectro de suelos en los que se puede medir a suelos con contenido orgánico o humus (Bobrov et al., 2019).

Para las medidas en sondas FDR y TDR, sabemos, la velocidad de viaje, $v$, de una onda electromagnética a través de un medio dieléctrico se puede calcular (1) como (Yu \& Yu, 2006):

$v=\frac{c}{\sqrt{K_{a}}}$

Donde: $c$ es la velocidad de la onda electromagnética en el espacio libre $\left(3 \times 10^{8} \mathrm{~m} / \mathrm{s}\right), \mathrm{K}_{\mathrm{a}}$ : constante dieléctrica aparente, el tiempo de viaje, $t$, en un medio con longitud, $L$, para la onda electromagnética está dado por (Yu \& Yu, 2006):

$t=\frac{2 L}{v}$

Expresando la constante dieléctrica en términos (1) y obtenemos:

$K_{a}=\left(\frac{c t}{2 L}\right)^{2}$

Si se define la longitud aparente $l_{a}$ como:

$$
l_{a}=\left(\frac{c t}{2}\right)^{2}
$$

la constante dieléctrica aparente puede expresarse en términos de la longitud aparente y la longitud del medio

$K_{a}=\left(\frac{l_{a}}{L}\right)^{2}$

En la señal TDR, la longitud aparente se determina a partir del análisis del tiempo transcurrido entre las reflexiones, tal como se observa en la Figura 4. 


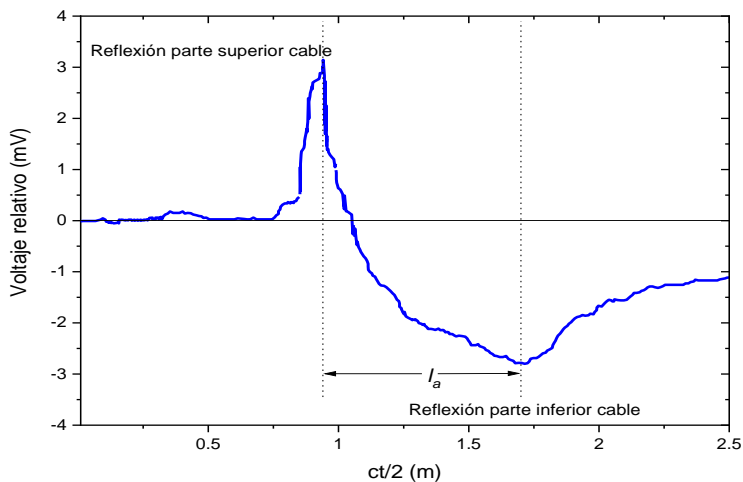

Figura 4. Reflexión de la señal en sonda TDR (Yu \& Yu, 2006)

El contenido volumétrico de agua, $\theta$, es porcentaje volumétrico del volumen total del suelo. El contenido másico de agua, w, es el porcentaje másico de agua al suelo seco sólido estas variables están relacionadas

$\theta=w \frac{\rho_{d}}{\rho_{w}}$

donde $\rho_{d}$ y $\rho_{w}$ son la densidad de suelo seco y la densidad del agua respectivamente.

El contenido volumétrico de agua se relaciona con la constante dieléctrica aparente, $K_{a}$ del suelo con la ecuación de tercer grado (7) propuesta por Topp (Topp et al., 1980)

$$
\theta=4.3 * 10^{-6} K_{a}^{3}-5.5 * 10^{-4} K_{a}^{2}+2.92 * 10^{-2} K_{a}-5.3 * 10^{-2}
$$

Esta ecuación se resolvió por Dey y colaboradores 2015 para varios contenidos de humedad del suelo obteniendo diferentes valores de constante dieléctrica aparente o permitividad relativa $\varepsilon \tau$ Tabla 4, a una frecuencia de $10 \mathrm{GHz}$.

Tabla 4. Permitividad relativa del suelo (Dey, 2015)

\begin{tabular}{c|c}
\hline Condición del suelo & Permitividad $K_{a}$ \\
\hline Seco & 2.53 \\
& \\
Húmedo $2.18 \%$ & 2.5 \\
Húmedo $3.88 \%$ & 3.6 \\
Húmedo $18.8 \%$ & 13 \\
\hline
\end{tabular}

Esta variación hace que la fluctuación de amplitud TDR indique el cambio en el contenido de humedad del suelo, (S.U. et al., 2014), (Dey et al., 2015). La expresión general para la permitividad está dada por (8):

$$
K^{*}=K^{\prime}+j\left\{K^{\prime \prime}+\left(\frac{\sigma_{d c}}{\omega \varepsilon_{0}}\right)\right\}
$$

Donde: $K^{*}$ constante dieléctrica compleja, $K^{\prime}$ parte real, $K^{\prime \prime}$ parte imaginaria, $\sigma_{d c}$ conductividad a frecuencia cero, $\omega$, frecuencia angular, $\varepsilon_{0}$ permitividad en el vacío $(8.85 \times$ $10^{-12} \mathrm{~F} / \mathrm{m}$ )

Se determinó el efecto de la densidad aparente, la temperatura y el contenido de humedad soluble, encontrando que la parte real $K^{\prime}$ de la permitividad compleja es independiente de la frecuencia, pero es altamente sensible al contenido de humedad del suelo. Topp y colaboradores (Topp et al., 1980) han estudiado la relación entre el contenido de humedad volumétrico y la constante dieléctrica para el rango de frecuencias de $1 \mathrm{MHz}$ a 1 GHz., la influencia de varias características del suelo en el contenido volumétrico, textura, estructura, contenido de humedad, temperatura, densidad a frecuencias entre $1 \mathrm{~Hz}$ a $1 \mathrm{Gz}$ encontrando que tienen poco efecto sobre el valor de $K_{a}$. Se ha propuesto una ecuación generalizada por Bhat y coautores 2007 para mejorar su aplicabilidad a todo tipo de suelo y un amplio rango de frecuencia, teniendo en cuenta la composición de los minerales del suelo (9)

$$
\begin{aligned}
& \sqrt{k}=\left[(1-\eta)\left(M_{1} * \sqrt{k_{M_{1}}}+M_{2} * \sqrt{k_{M_{2}}}\right)+\eta *\left(S_{r} * \sqrt{k_{P F_{1}}}+\left(1-S_{r}\right) *\right.\right. \\
& \left.\left.\sqrt{k_{P F_{2}}}\right)\right]
\end{aligned}
$$

Donde $\quad M_{1}$ y $M_{2}$ composición porcentual de los minerales, $k_{M_{1}}$ y $k_{M_{2}}$ constantes dieléctricas de los minerales, $k_{P F_{1}}$ y $k_{P F_{2}}$ constantes dieléctricas de los poros y fluidos, $\eta$ porosidad, y $S_{r}$ grado de saturación del material.

Otro método para mejorar el rango de frecuencia de medición utiliza la técnica de muestreo de tiempo equivalente reportada por Sulthoniy y Rizqulloh (Sulthoni \& Rizqulloh, 2019) con un sensor de bajo costo, que se basa en la medición de la velocidad de propagación de una onda electromagnética en el suelo. La señal de entrada es un pulso de señal rectangular de ciclo del $50 \%$ y la señal de salida tiene la forma especificada en la Figura 5 que puede ser positiva o negativa dependiendo del desfase en la impedancia.

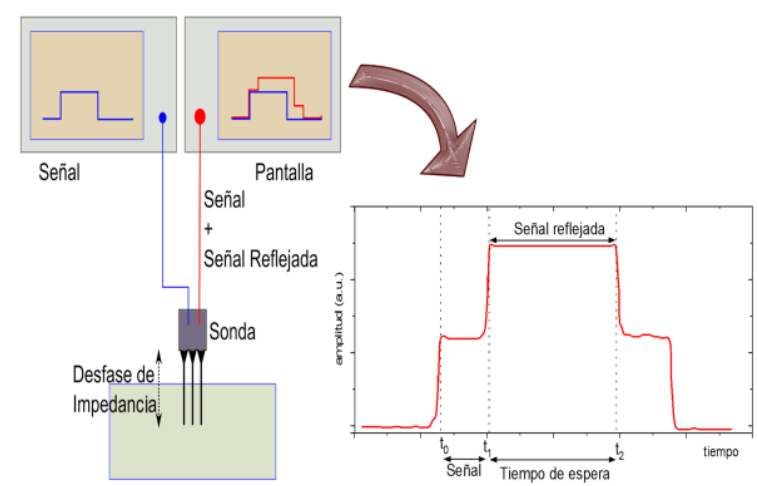

Figura 5. Diagrama de una sonda TDR y su señal de entrada y salida (Sulthoni \& Rizqulloh, 2019)

Usando la ecuación (2) se puede calcular la velocidad de propagación de la onda a partir del tiempo de recorrido $(t)$ y la longitud de la sonda $(l)$

$$
v=\frac{2 l}{t}
$$

La velocidad del pulso cambia con el contenido de humedad del suelo por el valor alto de la constante dieléctrica del agua. La velocidad del pulso en la sonda se mide y se correlaciona con la humedad del suelo. Una velocidad baja indica mayor contenido de humedad. El tiempo de pausa $(\Delta t)$ se obtiene como:

$$
\Delta t=t_{1}-t_{2}=t_{3}-t_{4}
$$

Y el contenido de humedad es calculada con en Topp et. al.(Topp et al., 1980):

$\theta=4.3 * 10^{-6} K_{a}^{3}-5.5 * 10^{-4} K_{a}^{2}+2.92 * 10^{-2} K_{a}-5.3 * 10^{-2}$

donde $K_{a}=0.75 \Delta t$.

\section{7. (FDR)-Técnica capacitiva y técnica de reflectometría de dominio de frecuencia.}

El principio de reflectometría del dominio de la frecuenciaFDR (Frequency-Domain Reflectometry) se basa en el arreglo de un circuito oscilante y un sensor que está incrustado en el suelo. 
Esta técnica considera al suelo como el dieléctrico de un condensador, la constante dieléctrica del suelo varía en función de su contenido en agua, variando la capacitancia del condensador. Cuando este arreglo se integra en un circuito oscilador, las variaciones en la capacidad del condensador y la permitividad del medio conllevan a una variación en la frecuencia de la señal generada por el oscilador (Whalley et al., 1992), (M. Robinson \& Dean, 1993), (Gardner et al., 1998). A partir de este principio se pueden mencionar dos tipos de técnicas bajo la denominación FDR: los sensores capacitivos y los equipos FDR.

En los sensores capacitivos la permitividad del medio se determina midiendo el tiempo de carga del condensador. Por su parte, los FDR realizan un barrido de frecuencia dentro de un determinado rango y obtienen la frecuencia de resonancia, que se relaciona con el CHS.

Las sondas de estos equipos suelen presentarse en tres formas diferentes: placas planas, barras cilíndricas y anillos metálicos alrededor de un cilindro, Figura 6. En los sensores FDR con la configuración en anillo, la sonda se introduce dentro de un tubo de acceso ya instalado en el suelo para colocar varios sensores que puedan realizar medidas a diferentes profundidades. Avances tecnológicos en este tipo de sensores han servido para su miniaturización y/o análisis del CHS en el lugar de trabajo, además de mejores métodos de calibración con mayor exactitud y rapidez (Farahani et al., 2014).

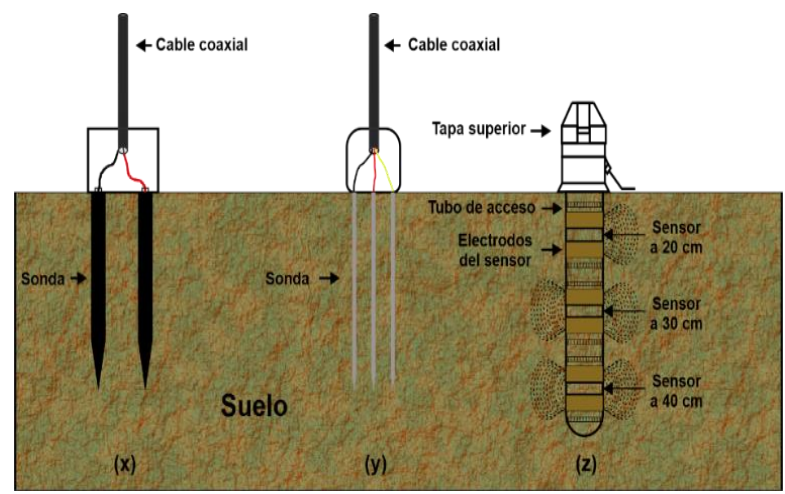

Figura 6. Tipos de sondas FDR. (x): de placas planas, (y) de barras cilíndricas, (z): de anillos metálicos alrededor de un cilindro (Kargas \& Soulis, 2012).

Los sensores capacitivos y FDR operan a frecuencias comprendidas entre 10 y $150 \mathrm{MHz}$ (S.U. et al., 2014), (Kargas \& Soulis, 2012), utilizando frecuencias de $500 \mathrm{MHz}$ es posible determinar la salinidad del suelo (Skierucha \& Wilczek, 2010).

La permitividad relativa está representada por un numero complejo y en algunas investigaciones (S.U. et al., 2014), (Topp et al., 1980), (Skierucha \& Wilczek, 2010) sostienen que variables como la temperatura o la salinidad influyen en el valor de la componente imaginaria de la permitividad relativa, ocasionando variaciones en la medida del contenido de agua del suelo. La parte real, sin embargo, es independiente de la temperatura y de la frecuencia de trabajo.

\subsection{Cálculo del CHS}

En (Böhme et al., 2013) utilizaron el sensor PR2 de Delta Devices Ltd (Delta-T Devices Ltd, 2016) para el monitoreo de la humedad del suelo. Este sensor está diseñado para realizar lecturas portátiles desde un tubo de acceso simultáneamente a cuatro profundidades hasta $40 \mathrm{~cm}$. La sonda de perfil consta de una varilla sellada de policarbonato de $25 \mathrm{~mm}$ de diámetro, con sensores electrónicos dispuestos a intervalos fijos a lo largo de su longitud. La sonda crea una señal de $100 \mathrm{MHz}$ que se transmite como un campo electromagnético que se extiende unos $100 \mathrm{~mm}$ en el suelo. El CHS $\left(\theta_{V}\right)$ se puede predecir a partir de la raíz cuadrada de la permitividad eléctrica $\sqrt{\varepsilon}$ del volumen del suelo utilizando la siguiente ecuación:

$$
\sqrt{\varepsilon}=a_{0}+a_{1} \times \theta_{V}
$$

Donde $a_{0}$ es el desplazamiento del suelo y $a_{1}$ la pendiente. Para suelos con mineral generalizado ( $\mathrm{min}$ ), suelos orgánicos (org), el fabricante proporciona $a_{0}$ y $a_{1} \quad\left(a_{0}(\mathrm{~min})=1.6, a_{0}(\mathrm{org})=\right.$ 1.3, $\left.a_{1}(\min )=8.4, a_{1}(\mathrm{org})=7.7\right)$.

La relación entre la salida de voltaje de PR2 y $\sqrt{\varepsilon}$ esta ajustada por una ecuación polinomial (19) hasta un CHS de $0.5 \mathrm{~m}^{3} / \mathrm{m}^{3}$ :

$\sqrt{\varepsilon}=1.125-5.53 \times V+67.17 \times V^{2}-234.42 \times V^{3}+413.56 \times V^{4}-$

$356.68 \times V^{5}+121.53 \times V^{6}$

Combinando las ecuaciones (13) y (14) resulta en una conversión polinomial (ecuación (15)) para el cálculo del CHS $\left[\mathrm{m}^{3} / \mathrm{m}^{3}\right]$ : donde $a_{0}$ y $a_{1}$ son los coeficientes de calibración de la ecuación (13).

$\theta_{V}=\frac{\left(1.125-5.53 \times V+67.17 \times V^{2}-234.42 \times V^{3}+413.56 \times V^{4}-356.68 \times V^{5}+121.53 \times V^{6}-a_{0}\right)}{a_{1}}(15)$

Esta tecnología, al igual que el resto, presenta determinadas limitaciones. La repetitividad de los sensores en la medida del contenido volumétrico de agua es mayor en suelos con un CHS inferior al 5\% y, por lo tanto, su sensibilidad se incrementa en condiciones de suelo relativamente seco (S.U. et al., 2014). Por otro lado, la sensibilidad se ve afectada por el valor de la densidad del suelo. Su dependencia de la textura del suelo supone la necesidad de llevar a cabo una calibración específica en cada caso.

\subsection{Impedancia eléctrica}

$\mathrm{Su}$ principio de funcionamiento utiliza la técnica de espectroscopia de impedancia aplicando una pequeña señal de potencial (V) a un electrodo y midiendo su respuesta en corriente (I) a diferentes frecuencias. Las mediciones de potencial a diversas frecuencias (tiempo y corriente) dan como resultado una impedancia, en donde se compara la amplitud y la fase de las señales incidentes y reflejadas desde el suelo cerca del sensor. Las mediciones de las partes reales e imaginarias de la impedancia del suelo a múltiples frecuencias le dan al sensor la capacidad de detectar variaciones en las concentraciones iónicas distintas del CHS (Báez et al., 2004).

Entre las distintas formas de medir la impedancia, una de ellas es el método convencional o estándar: el cual consiste en utilizar un circuito de tipo analógico o convertidor analógico-digital y un algoritmo de procesamiento de señales para analizar la respuesta final; dicho circuito es usado para medir el desplazamiento tanto de la amplitud como de la fase, o bien, las partes imaginarias y reales, que se obtienen al aplicar un voltaje senoidal puro a los electrodos del sensor.

Los sensores de impedancia se pueden clasificar según el material de que están hechos en tres clases: cerámico o semiconductor, sensores basados en polímeros orgánicos y 
sensores híbridos orgánicos e inorgánicos, y según el mecanismo del transporte eléctrico en dos más: dispositivos sensores electrónicos y de conducción iónica (Farahani et al., 2014).

\section{Cálculo del CHS}

Se realiza utilizando la ecuación propuesta por Topp et al., 1980 (Eq. 7), y sigue iguales condiciones. Un análisis más profundo sobre el desarrollo matemático se encuentra en Rusu et al. 2019, Gaskin \& Miller 1996, Rinaldi \& Francisca, 1999, utiliza un arreglo particular donde un sensor miniaturizado, de baja potencia, en tiempo real, multiparámetro y de bajo costo, donde se mide el CHS utilizando dos frecuencias para medir la conductividad y el CHS respectivamente.

Tabla 6. Ventajas y desventajas de los sensores basados en las propiedades eléctricas (Realización propia)

\begin{tabular}{|c|c|c|}
\hline $\begin{array}{l}\text { Técnicas } \\
\text { eléctricas }\end{array}$ & Ventajas & Desventajas \\
\hline $\begin{array}{l}\text { Técnica de } \\
\text { sensor de } \\
\text { resistividad del } \\
\text { suelo }\end{array}$ & $\begin{array}{l}\text { Puede proporcionar un contenido } \\
\text { absoluto de agua en el suelo a } \\
\text { cualquier profundidad. Se puede } \\
\text { ajustar para tener mayor alcance } \\
\text { espacial. Un nivel de precisión } \\
\text { relativamente alto cuando la } \\
\text { concentración iónica del suelo no } \\
\text { cambia. Puede ser leído por } \\
\text { métodos } \\
\text { Facilidad de aplicación de la } \\
\text { técnica y de mejor costo con } \\
\text { relación a otras técnicas } \\
\text { conductivas. }\end{array}$ & $\begin{array}{l}\text { Requiere de calibración } \\
\text { previa y para los diferentes } \\
\text { tipos de suelos. La calibración } \\
\text { no es estable con el tiempo y } \\
\text { se ve afectada por la } \\
\text { concentración iónica. } \\
\text { El costo del equipo para } \\
\text { generar la señal y el sistema } \\
\text { de lectura es alto. }\end{array}$ \\
\hline $\begin{array}{l}\text { (TDR) - Técnica } \\
\text { de reflectometría } \\
\text { en el dominio } \\
\text { del tiempo }\end{array}$ & $\begin{array}{l}\text { Altos costos del equipo. Permite } \\
\text { el monitoreo y automatización de } \\
\text { la medida de humedad, método } \\
\text { rápido y repetible. Se puede } \\
\text { operar hasta una frecuencia de } 1 \\
\text { GHz. Es independiente de la } \\
\text { textura del suelo y la temperatura; } \\
\text { poco dependiente del contenido } \\
\text { de sales. Se ha modificado para } \\
\text { adaptarse a plataformas móviles, } \\
\text { como tractores, vehículos todo } \\
\text { terreno (ATV) o equipos de } \\
\text { pulverización. }\end{array}$ & $\begin{array}{l}\text { Alto costo inicial, existe } \\
\text { pérdida de reflexión en suelos } \\
\text { altamente salinos, aumento de } \\
\text { la conductividad con la } \\
\text { humectación de la masa del } \\
\text { suelo, lo que afecta las } \\
\text { medidas. Se pueden obtener } \\
\text { buenas mediciones en un } \\
\text { suelo salino muy seco en } \\
\text { comparación con los suelos } \\
\text { salinos húmedos. } \\
\text { Lleva mucho tiempo realizar } \\
\text { muchas mediciones } \\
\text { repetitivas. calibración } \\
\text { Requiere una con } \\
\text { frecuente durante la } \\
\text { implementación. }\end{array}$ \\
\hline $\begin{array}{l}\text { (FDR) - Técnica } \\
\text { capacitiva y } \\
\text { técnica de } \\
\text { reflectometría de } \\
\text { dominio de } \\
\text { frecuencia }\end{array}$ & $\begin{array}{l}\text { Alto costo inicial, pero menor que } \\
\text { el TDR, válido para contenidos de } \\
\text { humedad cercanas al } 50 \% \text { o } \\
\text { menores. }\end{array}$ & $\begin{array}{l}\text { Falla en el suelo expansivo } \\
\text { debido al agrietamiento del } \\
\text { suelo. } \\
\text { Requiere una calibración } \\
\text { frecuente durante la } \\
\text { implementación. }\end{array}$ \\
\hline $\begin{array}{l}\text { Impedancia } \\
\text { eléctrica }\end{array}$ & $\begin{array}{l}\text { Resultados rápidos y confiables. } \\
\text { Bajo costo en comparación con la } \\
\text { TDR, FDR y Técnica de } \\
\text { atenuación de rayos gamma. }\end{array}$ & $\begin{array}{l}\text { Requieren de calibración } \\
\text { previa y de personal } \\
\text { capacitado para la aplicación } \\
\text { de los ensayos. La estabilidad } \\
\text { a largo plazo es cuestionable. }\end{array}$ \\
\hline
\end{tabular}

\section{Conclusiones}

El suelo es un sistema trifásico compuesto por los minerales del suelo (sólidos), agua (líquido) y aire (gas), el CHS está presente como agua estructural, fisisorción, quimisorción gravitacional o atrapada en el aire en forma de vapor de agua. Las técnicas suelen diferir en el concepto de humedad, pero en general los resultados se refieren como humedad total.

Los parámetros que tienen influencia en el CHS son: la mineralogía, salinidad, porosidad, temperatura de medición, presencia de materia orgánica, estructura matricial del suelo, unidad del suelo entre otras.

El CHS es una parte primordial para los componentes vitales y afecta las propiedades mecánicas del suelo, tales como: la consistencia, la compatibilidad, el agrietamiento, la expansión, la contracción y la densidad real.

Existen diferentes técnicas para medir el CHS, la tendencia es hacia la automatización, buscando el monitoreo remoto, Las técnicas basadas en las propiedades eléctricas ofrecen bastantes ventajas con respecto a otras técnicas dado que son más económicas y existe una base teórica sólida, lo que permite el diseño y mejoramiento de los sensores.

Dependiendo de la cantidad de humedad contenida en el suelo se determinan las características que puede presentar. Las técnicas presentadas se han utilizado ampliamente y algunas aun teniendo en cuenta sus costos siguen siendo estudiadas, dado que poseen una gran precisión, son versátiles y de bajo costo, además permiten la toma de datos in situ.

El avance en el desarrollo de sensores más económicos y precisos ayudará a las economías basadas en la agricultura a tener datos que optimicen el uso del agua, y por tanto no entrar en competencia con el consumo humano. En países con un mayor crecimiento poblacional se verían altamente favorecidos por este tipo de módulos y sistemas. De igual manera ayudará a la prevención y manejo de catástrofes como medio de alerta para evitar los deslizamientos de tierra en topografías donde el grado de inclinación del suelo es crítico y en zonas de desarrollo humano, al ser determinante en la presencia de taludes y deslaves por inestabilidad del suelo.

También se ha examinado la importancia del CHS en otros campos, en los sistemas ambientales globales como factor de importancia en el calentamiento global, todos estos factores hacen que el análisis del CHS sea de gran importancia y por tanto se invierta un buen capital humano y esfuerzo económico en su determinación. Los métodos abarcados durante este trabajo no necesariamente son los mejores o superiores a otros, el objetivo de este estudio es el conocer cada uno, su forma de operación, las matemáticas en las que se basan, y dependiendo del trabajo a realizar, uno será más ventajoso que el otro.

\section{Referencias}

Adla, S., Rai, N. K., Karumanchi, S. H., Tripathi, S., Disse, M., \& Pande, S. (2020). Laboratory Calibration and Performance Evaluation of Low-Cost Capacitive and Very Low-Cost Resistive Soil Moisture Sensors. Sensors, 20(2), 363. DOI: 10.3390/s20020363

Báez, S., Tristancho, J. L., Peña, D. Y., Vázquez, C., \& Anaya, H. A. (2004). La espectroscopia de impedancia electroquímica (EIS) aplicada al estudio del mecanismo de la corrosión en caliente por sales fundidas. Dyna, 71(144), 39-47.

Berney, E. S., \& Kyzar, J. D. (2012). Evaluation of Nonnuclear Soil Moisture and Density Devices for Field Quality Control. Transportation Research Record: Journal of the Transportation Research Board, 2310(1), 18-26. DOI: $10.3141 / 2310-03$

Bhat, A. M., B, H. R., \& Singh, D. N. (2007). A Generalized Relationship for Estimating Dielectric Constant of Soils. Journal of ASTM International, 4(7), 1-17. DOI: 10.1520/JAI100595

Bobrov, P. P., Belyaeva, T. A., Kroshka, E. S., \& Rodionova, O. V. (2019). Soil Moisture Measurement by the Dielectric Method. Eurasian Soil Science, 52(7), 822-833. DOI: 10.1134/S106422931905003X

Böhme, B., Becker, M., \& Diekkrüger, B. (2013). Calibrating a FDR sensor for soil moisture monitoring in a wetland in Central Kenya. Physics and Chemistry of the Earth, Parts A/B/C, 66, 101-111. DOI: $10.1016 /$ j.pce.2013.09.004

Cruz, A. B., Barra, J. E., Castillo, R. F. del, \& Gutiérrez, C. (2004). La calidad del suelo y sus indicadores. Ecosistemas, 13(2), Article 2. DOI: $10.7818 /$ ECOS.572 
Delta-T Devices Ltd. (2016). User Manual for the Profile Probe typePR2. https://delta-t.co.uk/wpcontent/uploads/2017/02/PR2_user_manual_version_5.0.pdf

Dey, S., Kalansuriya, P., \& Karmakar, N. C. (2015). A novel time domain reflectometry based chipless RFID soil moisture sensor. 2015 IEEE MTTS International Microwave Symposium, 1-4. DOI: 10.1109/MWSYM.2015.7166925

Dobriyal, P., Qureshi, A., Badola, R., \& Hussain, S. A. (2012). A review of the methods available for estimating soil moisture and its implications for water resource management. Journal of Hydrology, 458-459, 110-117. DOI: $10.1016 /$ j.jhydrol.2012.06.021

Domingo-Santos, J., Villarán, R., Corral-Pazos-de-Provens, E., \& RappArrarás, Í. (2008). Estimación de la capacidad de retención de agua en el suelo: Revisión del parámetro CRA. Investigación agraria. Sistemas y recursos forestales, ISSN 1131-7965, Vol. 15, $N^{o} 1$, 2006, pags. 14-23, 15.

Farahani, H., Wagiran, R., \& Hamidon, M. (2014). Humidity Sensors Principle, Mechanism, and Fabrication Technologies: A Comprehensive Review. Sensors, 14(5), 7881-7939. DOI: 10.3390/s140507881

Florentino, A. (2006). Métodos para medir el contenido de agua en el suelo. Venesuelos, 14(1), 48-70.

Fraden, J. (2016). Humidity and Moisture Sensors. En J. Fraden, Handbook of Modern Sensors (pp. 507-523). Springer International Publishing. DOI: $10.1007 / 978-3-319-19303-8 \_14$

Gardner, C. M. K., Dean, T. J., \& Cooper, J. D. (1998). Soil Water Content Measurement with a High-Frequency Capacitance Sensor. Journal of Agricultural Engineering Research, 71(4), 395-403. https://doi.org/10.1006/jaer.1998.0338

Gaskin, G. J., \& Miller, J. D. (1996). Measurement of Soil Water Content Using a Simplified Impedance Measuring Technique. Journal of Agricultural Engineering Research, 63(2), 153-159. https://doi.org/10.1006/jaer.1996.0017

Guzzetti, F., Gariano, S. L., Peruccacci, S., Brunetti, M. T., Marchesini, I., Rossi, M., \& Melillo, M. (2020). Geographical landslide early warning systems. Earth-Science Reviews, 200, 102973. https://doi.org/10.1016/j.earscirev.2019.102973

Juárez Badillo, E., \& Rico Rodríguez, A. (2010). Mecánica de suelos. Limusa : Noriega.

Kargas, G., \& Soulis, K. X. (2012). Performance Analysis and Calibration of a New Low-Cost Capacitance Soil Moisture Sensor. Journal of Irrigation and Drainage Engineering, 138(7), 632-641. https://doi.org/10.1061/(ASCE)IR.1943-4774.0000449

Liu, L., Estiarte, M., \& Peñuelas, J. (2019). Soil moisture as the key factor of atmospheric $\mathrm{CH} 4$ uptake in forest soils under environmental change. Geoderma, 355 ,

113920 . https://doi.org/10.1016/j.geoderma.2019.113920

Motsara, M. R., \& Roy, R. N. (2008). Guide to laboratory establishment for plant nutrient analysis. Food and Agriculture Organization of the United Nations.

Muñoz-Carpena, R. (2004). Field devices for monitoring soil water content. EDIS, 2004(8).

Rêgo Segundo, A., Martins, J., Monteiro, P., de Oliveira, R., \& Freitas, G. (2015). A Novel Low-Cost Instrumentation System for Measuring the Water Content and Apparent Electrical Conductivity of Soils. Sensors, 15(10), 25546-25563. https://doi.org/10.3390/s151025546

Rinaldi, V. A., \& Francisca, F. M. (1999). Impedance Analysis of Soil Dielectric Dispersion (1 MHz-1 GHz). Journal of Geotechnical and Geoenvironmental Engineering, 125(2), 111-121. https://doi.org/10.1061/(ASCE)1090-0241(1999)125:2(111)

Robinson, D. A., Campbell, C. S., Hopmans, J. W., Hornbuckle, B. K., Jones, S. B., Knight, R., Ogden, F., Selker, J., \& Wendroth, O. (2008). Soil Moisture Measurement for Ecological and Hydrological Watershed-Scale Observatories: A Review. Vadose Zone Journal, 7(1), 358-389. https://doi.org/10.2136/vzj2007.0143
Robinson, M., \& Dean, T. J. (1993). Measurement of near surface soil water content using a capacitance probe. Hydrological Processes, 7(1), 77-86. https://doi.org/10.1002/hyp.3360070108

Robock, A., Vinnikov, K. Y., Srinivasan, G., Entin, J. K., Hollinger, S. E., Speranskaya, N. A., Liu, S., \& Namkhai, A. (2000). The Global Soil Moisture Data Bank. Bulletin of the American Meteorological Society, 81(6), 1281-1299.

Rusu, C., Krozer, A., Johansson, C., Ahrentorp, F., Pettersson, T., Jonasson, C., Rösevall, J., Ilver, D., Terzaghi, M., Chiatante, D., \& Montagnoli, A. (2019). Miniaturized wireless water content and conductivity soil sensor system. Computers and Electronics in Agriculture, 167, 105076. https://doi.org/10.1016/j.compag.2019.105076

Schmugge, T. J., Jackson, T. J., \& McKim, H. L. (1980). Survey of methods for soil moisture determination. Water Resources Research, 16(6), 961979. https://doi.org/10.1029/WR016i006p00961

Seneviratne, S. I., Corti, T., Davin, E. L., Hirschi, M., Jaeger, E. B., Lehner, I., Orlowsky, B., \& Teuling, A. J. (2010). Investigating soil moistureclimate interactions in a changing climate: A review. Earth-Science $\begin{array}{lll}\text { Reviews, } & \text { 99(3-4), 125-161. }\end{array}$ https://doi.org/10.1016/j.earscirev.2010.02.004

Sinha, S., Norouzi, A., Pradhan, A., Yu, X., Seo, D.-J., \& Zhang, N. (2017). A Field Soil Moisture Study Using Time Domain Reflectometry (TDR) and Time Domain Transmissivity (TDT) Sensors. DEStech Transactions on Materials Science and Engineering, ictim. https://doi.org/10.12783/dtmse/ictim2017/10109

Skierucha, W., \& Wilczek, A. (2010). A FDR Sensor for Measuring Complex Soil Dielectric Permittivity in the $10-500 \mathrm{MHz}$ Frequency Range. Sensors, 10(4), 3314-3329. https://doi.org/10.3390/s100403314

S.U., S. L., Singh, D. N., \& Shojaei Baghini, M. (2014). A critical review of soil moisture measurement. Measurement, 54, 92-105. https://doi.org/10.1016/j.measurement.2014.04.007

Sulthoni, M. A., \& Rizqulloh, M. A. (2019). A Low Cost Microcontrollerbased Time Domain Reflectometer for Soil Moisture Measurement. 2019 International Conference on Electrical Engineering and Informatics (ICEEI), 197-200. https://doi.org/10.1109/ICEEI47359.2019.8988875

Topp, G. C., Davis, J. L., \& Annan, A. P. (1980). Electromagnetic determination of soil water content: Measurements in coaxial transmission lines. Water Resources Research, 16(3), 574-582. https://doi.org/10.1029/WR016i003p00574

van Dam, R. L., Borchers, B., \& Hendrickx, J. M. H. (2005). Methods for prediction of soil dielectric properties: A review (R. S. Harmon, J. T. Broach, \& J. H. Holloway, Jr., Eds.; p. 188). https://doi.org/10.1117/12.602868

Verwey, E. J. W. (1947). Theory of the Stability of Lyophobic Colloids. The Journal of Physical and Colloid Chemistry, 51(3), 631-636. https://doi.org/10.1021/j150453a001

Walker, J. P., Willgoose, G. R., \& Kalma, J. D. (2004). In situ measurement of soil moisture: A comparison of techniques. Journal of Hydrology, 293(1-4), 85-99. https://doi.org/10.1016/j.jhydrol.2004.01.008

Whalley, W. R., Dean, T. J., \& Izzard, P. (1992). Evaluation of the capacitance technique as a method for dynamically measuring soil water content. Journal of Agricultural Engineering Research, 52, 147-155. https://doi.org/10.1016/0021-8634(92)80056-X

Yu, X., \& Yu, X. (2006). Time Domain Reflectometry Tests of Multilayered Soil. 17.

Zazueta, F. S., \& Xin, J. (1994). Soil Moisture Sensors. 11. 